\title{
CEBRAS: CROSS ELEVATION BEAM RANGE AMBIGUITY SUPPRESSION FOR HIGH-RESOLUTION WIDE-SWATH AND MIMO-SAR IMAGING
}

\author{
G. Krieger, S. Huber, M. Villano, M. Younis, T. Rommel, P. Lopez Dekker, F. Queiroz de Almeida, A. Moreira \\ Microwaves and Radar Institute, German Aerospace Center (DLR)
}

\begin{abstract}
In this paper we propose a new hybrid technique to suppress range ambiguities in spaceborne SAR systems with multiple elevation beams. First, conventional scan-on-receive (SCORE) is performed in real-time onboard the satellite by employing a set of dispersive beams that maximize the collected signal energy for each transmitted pulse. The range ambiguities are then removed in a second step by a joint processing of the signals collected by the multiple elevation beams. The suggested two-stage approach has the advantage that a more robust range ambiguity suppression, which may involve advanced nulling techniques to account for local topography as well as satellite attitude and instrument phase errors, can be performed on ground without tremendously increasing the onboard processing demands or the data downlink volume.
\end{abstract}

Index Terms - Range Ambiguities, MIMO-SAR, Digital Beamforming, Multiple Elevation Beams, HRWS

\section{INTRODUCTION}

A major challenge for the implementation of multiple swath and staggered SAR systems is the suppression of range ambiguities [1]-[4]. This becomes evident from Figure 1, which shows in black the wide transmit antenna pattern that is obtained by simultaneously activating all feed elements of a reflector-based SAR system like Tandem-L [9], [10]. The narrow colored patterns show in contrast, for one instant of time, the receive beams that simultaneously map five subswaths with maximum gain. The dissociation between the widths of the transmit and receive beams has a severe consequence for the suppression of range ambiguities. While a classical SAR with a single elevation beam typically benefits from both the transmit and receive antenna patterns for range ambiguity suppression, a multiple elevation beam SAR has to rely on the receive beams alone. This is due to the fact that the desired radar echo and the most prominent range ambiguities are typically illuminated by almost the same transmit pattern. A very similar challenge arises for MIMO-SAR systems that employ subpulses or the generalized short-term shift-orthogonal waveforms [5]-[8]. To improve range ambiguity suppression, one has therefore to decrease the width and/or sidelobes of the Rx beams in elevation, which is typically associated with an increased antenna height.

Another option is the use of on-board null-steering techniques like the linear constrained minimum variance (LCMV) beamforming analyzed in [9] for the case of an array-fed reflector antenna. A similar approach was developed for planar arrays to separate the sub-pulses of a MIMO-SAR [7]. Real-time null steering in elevation is,

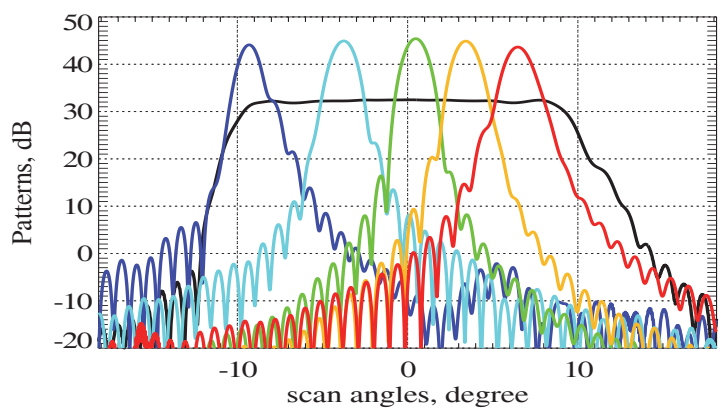

Figure 1: Antenna patterns for an L-band reflector-based SAR system with multiple feed elements in elevation. The broad transmit pattern (black) is obtained by activating all feed elements in elevation, while the five exemplary receive beams are steered to the expected directions of the simultaneously arriving radar echoes from five swaths.

however, extremely challenging since it is very sensitive to phase and amplitude offsets between the individual $\mathrm{Rx}$ channels as well as differences, frequency dependencies and mutual coupling between the individual element patterns. Furthermore, very accurate a priori knowledge of the topography and satellite attitude are required in real time to steer the narrow nulls to the correct elevation angles.

An alternative is to downlink the signals from all antenna elements and to perform the beamforming $a$ posteriori on the ground [1], [2], [5], [8]. This is, however, typically associated with an increased data rate, especially if a large antenna is used to obtain a high $\mathrm{Rx}$ gain over a wide swath. In the future, one could also think about adaptive real-time techniques [1], [11], but these will nevertheless significantly increase the on-board processing effort.

\section{A CLOSER LOOK TO NULL-STEERING}

\subsection{Range Migration and 2-D Antenna Patterns}

All previously introduced null-steering techniques regard range ambiguity suppression as a one-dimensional beamforming problem that operates only in the elevation direction. This neglects, however, a possible mismatch between the position of the nulls in the 2-D antenna pattern and the corresponding arrival angles of the range ambiguous radar signals that are, in fact, extended in azimuth. Here, mismatches can arise from (1) range cell migration, (2) topography variations in azimuth, (3) the curved Earth surface, and (4) antenna patterns that are non-separable in azimuth and elevation, as it typically applies to nonrectangular array antennas and array-fed reflectors. 

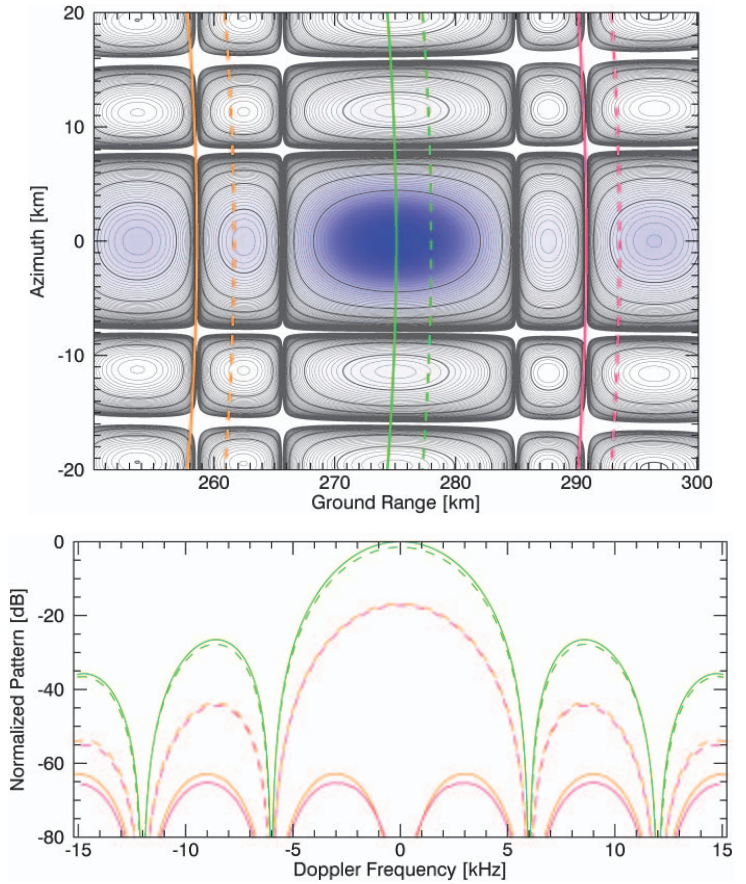

Figure 2: 2-D joint $T x$ and $R x$ antenna pattern of a rectangular array projected on a spherical Earth (top) and 1-D pattern cuts for fixed range bins corresponding to swath echoes and range ambiguities (bottom). An LCMV beam-former was employed to steer the nulls of a $2.5 \mathrm{~m} \times 2.5 \mathrm{~m} \mathrm{X}$-band array to the range ambiguous signals at zero-Doppler position (simulation parameters as in [5]). The desired swath echoes are shown in green, while the near and far range ambiguities are shown in orange and magenta, respectively. The dashed lines illustrate the effect of an increased topographic height of $1.5 \mathrm{~km}$.

To analyze these effects, we consider the radar echo of a single range bin. The location of the scatterers contributing to the radar echo at a given range can be computed from the intersection of the Earth surface and an ellipsoid whose focal points are the antenna positions during radar pulse transmission and reception (the ellipsoid collapses to a sphere in case of using the start-stop approximation). The contributions from individual scatterers are moreover weighted by the combined $\mathrm{Tx}$ and $\mathrm{Rx}$ antenna patterns which are specified in terms of their elevation and azimuth angles. Figure 2 and Figure 3 illustrate, for one instant of time, the weighting of the simultaneously arriving ground echoes for a planar array and an array-fed reflector SAR, where both employ an LCMV beamformer to steer nulls to the expected elevation angle of the range ambiguities.

Three important effects can be noted from these examples. First, even for flat topography and perfect nullsteering the range ambiguities do not completely vanish over the full Doppler band. This is due to the fact that the intersection of the isorange ellipsoid with the spherical Earth surface does not coincide with the position of the groundprojected pattern nulls. While the residual Dopplerdependent range ambiguities are small for the planar array,
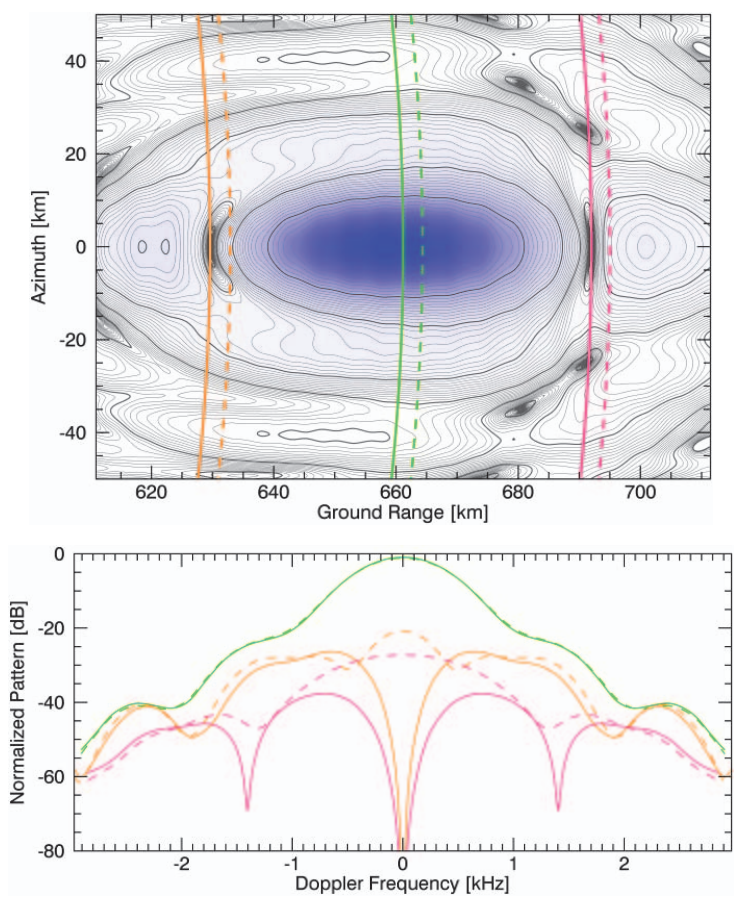

Figure 3: Antenna patterns as in Figure 2, but for an array-fed L-band reflector antenna with a diameter of $15 \mathrm{~m}$ steered in elevation to an off-boresight angle of $7.864^{\circ}$ (simulation parameters as in [10] for a PRI of $150 \mathrm{~ms})$. The dashed lines correspond to a height offset of $3 \mathbf{~ k m}$.

they may become notable if the reflector SAR in Figure 3 is operated in a fully polarimetric mode where a weak crosspol component has to compete against stronger co-pol returns from the ambiguous ranges. Second, a wrong topographic height (or satellite attitude) can significantly increase the range ambiguities. This effect is especially pronounced in the X-band example of Figure 2, where a height offset of $1.5 \mathrm{~km}$ raises the ambiguities to an unacceptable level of $-17 \mathrm{~dB}$. Note that even if the nullsteering had accounted for the topography at zero Doppler position, there may remain topographic variations within the azimuth antenna footprint that cannot be accounted for by null-steering in elevation. A stratovolcano like Mount Fuji has, e.g., on its upper western slopes height changes of 1.5 $\mathrm{km}$ over a distance of less than $3 \mathrm{~km}$, thereby making the surrounding plains prone to range ambiguities. A third observation is that the main beam gain is notably less affected by the assumed topographic errors $(1.5 \mathrm{~dB}$ and $0.18 \mathrm{~dB}$ loss for $\mathrm{X}$ - and L-band, respectively). This will become important for our further development in Section 3.

\subsection{Pulse Extension of Chirped Tx Signals}

Another challenge to real-time null-steering in elevation is the due consideration of both the pulse extension and the frequency dependence of the antenna patterns. To account for the extension of a chirped transmit pulse, it was proposed in [12] to add frequency dependent phase shifters within each elevation channel of a planar array. For a fixed 
frequency, the phase shifts vary linearly across the channels in elevation. This leads to a dispersive beam where the pattern maximum points, for each range frequency, towards a different elevation angle. For a flat Earth, the required phase shifts within the individual elevation channels can moreover be approximated by linear phase ramps, which allow an implementation of the frequency dependent phase shifters by mere time delays [12]. A similar strategy has been proposed in [7] to account for the extension of chirped Tx pulses in case of LCMV null-steering in elevation. The implementation of this technique relies, however, on the assumption that the relation between time and direction of arrival (ToA/DoA) is linear. Topographic changes within the swath and deviations from the linear ToA-DoA model for extended swath widths will therefore lead to a mispointing between the narrow nulls of the LCMV beamformer and the direction of arrival of the range ambiguities. While smooth deviations from the linear ToADoA model could probably be accounted for by slowly varying the time delays of the individual elevation channels in [7], it seems to be more difficult to account for fast topographic changes in range, not to speak about the azimuth dependence discussed in the previous section.

\section{CROSS ELEVATION BEAM RANGE AMBIGUITY SUPPRESSION (CEBRAS)}

\subsection{Basic Concept}

Essentially, two techniques have been devised for range ambiguity suppression in SAR systems with multiple elevation beams: real-time on-board null-steering [1], [7], [9] or downlink of all array element signals for a more sophisticated a posteriori processing on the ground [1], [2], [8]. The former technique suffers from a poor performance in case of topography as well as attitude and calibration errors, while the latter approach is typically associated with a high data volume that has to be transferred to the ground.

As an alternative to these techniques, we propose a hybrid approach that combines real-time beamforming onboard the satellite with an integrated array and SAR processing on the ground [5]. For this, we recall from Section 2 the striking difference between the performance of range ambiguity suppression and the gain loss of the main lobe in case of topographic errors. The same dissociation applies, in principle, also to the impact of satellite attitude and calibration errors. It is, therefore, promising to separate the more robust process of collecting maximum signal energy of the desired swath echoes by a conventional onboard beamformer [12] from the error-prone process of range ambiguity suppression. For the latter, we note that the most annoying range ambiguities for one imaging beam are, in fact, the desired signals of the other imaging beams that map the other sub-swaths (or follow the other subpulses in a MIMO-SAR). Since the signals from all imaging beams are anyway downloaded to the ground to map multiple subswaths, it is possible to a posteriori use the additional crossbeam information to mutually suppress range ambiguities.

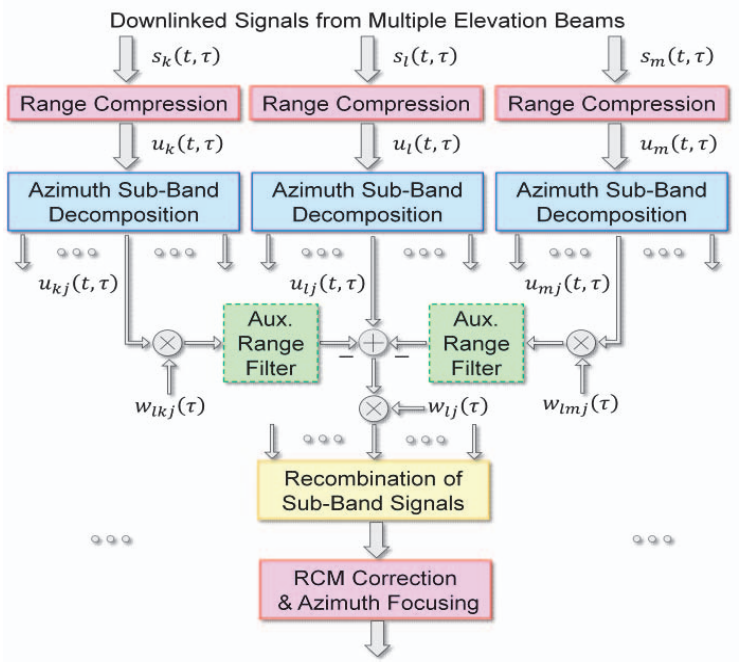

Figure 4: Flow chart of ambiguity suppression with CEBRAS.

\subsection{Processing Overview}

In its most simple form, range ambiguities for one beam can be mitigated by subtracting weighted signals from the other beams. From the discussion in Section 2.1 it is, however, clear that the range ambiguities may be characterized by azimuth spectra that have significantly different shapes if compared to those of the main beam spectra. Moreover, these differences can strongly depend on the local topography. To account for this effect, we decompose the range compressed signal $u_{k}(t, \tau)$ of each elevation beam $k$ into multiple Doppler sub-bands ${ }^{1}$ (or looks) $u_{k j}(t, \tau)$, where $t$ and $\tau$ refer to azimuth and range time, respectively. This azimuth decomposition is shown in the upper part of the flow chart in Figure 4. The next step is a weighted superposition of the beam signals from each sub-band $j$ with

$$
w_{l k j}(\tau)=\frac{A_{l}\left(\theta\left(\tau-\Delta \tau_{l k}, f_{j}\right), \varphi\left(\tau-\Delta \tau_{l k}, f_{j}\right) ; \tau\right)}{A_{k}\left(\theta\left(\tau, f_{j}\right), \varphi\left(\tau, f_{j}\right) ; \tau\right)}
$$

where $w_{l k j}(\tau)$ denotes the signal weighting from beam $k$ contributing to beam $l$. Here, $A_{i}(\theta, \varphi ; \tau)$ denotes the antenna pattern of beam $i$ at range time $\tau$. The angular coordinates $\theta$ and $\varphi$ are derived from the range echo time $\tau$, the transmit pulse separation $\Delta \tau_{l k}$, the Doppler subband frequency $f_{j}$, and the imaging geometry using a digital elevation model and estimates of the satellite's attitude and position parameters.

The dashed green boxes indicate an auxiliary spacevariant range filter to account for the case where the dispersive beams from Section 2.2 do not fully compensate the pulse extension effects in the real-time beamsteering. This causes essentially a nonuniform range spectrum for a fixed point on the ground [13]. Since the shapes of the main and ambiguous signal spectra can differ in this case, they should be compensated by an optional range variant filter.

\footnotetext{
${ }^{1}$ For staggered SAR [4] the azimuth samples of the range ambiguities are uncorrelated since they originate for each pulse from a different range. The frequency decomposition is therefore not applicable in this case.
} 
After the weighted addition of the cross-beam signals, a further weighting (or even space-variant range filter in case of applying the auxiliary filters) is required to compensate for the fact that the cross-beam signals may contain, besides scaled copies of the range ambiguous signals, also small copies of the desired main beam signal. This could introduce a phase and amplitude distortion within the considered Doppler band and has therefore to be compensated by applying the complex weights $w_{l j}(\tau)$. Thereafter, the full Doppler band is recovered by recombining the sub-band signals for each beam. The final processing steps are equal to those of conventional SAR focusing algorithms.

\subsection{Antenna Calibration / Estimation of Weights}

A critical issue is the provision of the complex weights $w_{l k j}(\tau)$. Here, three strategies can be followed. The first assumes that the antenna patterns can be provided with sufficient accuracy by an appropriate antenna model. This approach was implicitly assumed in most of the previously suggested algorithms for range ambiguity suppression.

An alternative are dedicated calibration data takes. For this, one may, e.g., use a long unambiguous PRI (or a single sub-pulse in case of a MIMO-SAR). By adjusting the individual trigger times of the multiple beam steering laws one can easily obtain estimates of the (complex) ratios between the sidelobes in one beam and the main lobes in the other beams as required for the estimate of $w_{l k j}(\tau)$ in (1).

A third more advanced approach is the estimation of the weighting factors directly from the recorded signals. This problem is closely related to the "cocktail party problem" (i.e. blind source/signal separation) where $N$ complex source signals $\left\{s_{1}(t), \ldots, s_{N}(t)\right\}$ are linearly mixed according to

$$
\left[\begin{array}{c}
u_{1}(t) \\
\vdots \\
u_{N}(t)
\end{array}\right]=\left[\begin{array}{ccc}
a_{11} & \cdots & a_{1 N} \\
\vdots & \ddots & \vdots \\
a_{N 1} & \cdots & a_{N N}
\end{array}\right] \cdot\left[\begin{array}{c}
s_{1}(t) \\
\vdots \\
s_{N}(t)
\end{array}\right]+\left[\begin{array}{c}
n_{1}(t) \\
\vdots \\
n_{N}(t)
\end{array}\right]
$$

where $\left\{u_{1}(t), \ldots, u_{N}(t)\right\}$ are the observed signals, $a_{i j} \in \mathbb{C}$ complex mixing factors, and $\left\{n_{1}(t), \ldots, n_{N}(t)\right\}$ additional noise. A wide class of algorithms, which basically exploit the statistical independence between, as well as second- and higher-order structure within the source signals, have been devised to recover the complex mixing factors and source signals from the observed signals [14]. This estimation process should be performed separately for each sub-look, and it should be repeated for each range bin. Knowing that the antenna patterns (and terrain topography) change smoothly among the range bins can further constrain the problem and increase the robustness of the estimation process. Moreover, a digital elevation model can be used for a precise location of the range ambiguities, which helps to establish a consistent data base of complex weighting coefficients (or to establish the associated antenna model). The robustness of the estimation process can be increased by a prior azimuth focusing of each range-ambiguous signal of the main beam and the corresponding cross-beam signal sub-looks. This provides more distinct non-Gaussian statistics for blind source separation.

\section{DISCUSSION}

An advanced range ambiguity suppression technique has been suggested that exploits the mutual information of multiple elevation beams that simultaneously scan the radar echoes arriving from different ranges. A critical component is the precise knowledge of the complex weighting coefficients (or antenna patterns). This information can be provided by (1) an antenna model, (2) appropriate calibration sequences or (3) directly from the recorded data. The latter approach leads to an adaptive range ambiguity suppression, which had, in much simpler form, already been suggested in [1] by exploiting the covariances between the signals of the different elevation beams. These second-order correlation based techniques are, however, insufficient to unambiguously estimate the mixing coefficients. This can easily be understood by noting that the number of unknown mixing coefficients exceeds the number of available covariance estimates. The problem can, however, be solved by employing higher-order statistics and/or exploiting nonstationarities in the acquired SAR data. A more detailed discussion will be provided in a follow-on paper.

\section{REFERENCES}

[1] H. Griffiths, P. Mancini, "Ambiguity Suppression in SARs Using Adaptive Array Techniques," Proc. IGARSS, pp. 1015-1018, 1991.

[2] G. Callaghan, I. Longstaff, "Wide-Swath Space-Borne SAR Using a Quad-Element Array,” IEE Proc. Radar Sonar Navig., Vol. 146, 1999.

[3] G. Krieger, I. Hajnsek, K. Papathanassiou, M. Younis, A. Moreira, "Interferometric Synthetic Aperture Radar (SAR) Missions Employing Formation Flying," Proc. IEEE, Vol. 98, pp. 816-843, May 2010.

[4] M. Villano, G. Krieger, A. Moreira, "Staggered SAR: High-Resolution Wide-Swath Imaging by Continuous PRI Variation", IEEE Trans. Geosci. Remote Sens., Vol. 52, pp. 4462-4479, 2014.

[5] G. Krieger, N. Gebert, A. Moreira, "Multidimensional Waveform Encoding: A New Digital Beamforming Technique for Synthetic Aperture Radar Remote Sensing," IEEE Trans. Geosci. Remote Sens., Vol. 46, pp. 31-46, 2008.

[6] G. Krieger, "MIMO-SAR: Opportunities and Pitfalls", IEEE Trans. Geoci. Remote Sens., Vol. 52, pp. 2628-2645, 2014.

[7] F. Feng, S. Li, W. Yu, P. Huang, W. Xu, "Echo Separation in Multidimensional Waveform Encoding SAR Remote Sensing Using an Advanced Null-Steering Beamformer," IEEE Trans. Geosci. Remote Sens., Vol. 50, pp. 4157-4172, 2012.

[8] F. He, X. Ma, Z. Dong, D. Liang, "Digital Beamforming on Receive in Elevation for Multidimensional Waveform Encoding SAR Sensing," IEEE Geosci. Remote Sens. Letters, Vol. 11, pp. 2173-2177, 2014.

[9] S. Huber, M. Younis, A. Patyuchenko, G. Krieger, A. Moreira, "Spaceborne Reflector SAR Systems with Digital Beamforming", IEEE Trans. Aerospace Electronic Systems, Vol. 48, No. 2, 2012.

[10] A. Moreira et al., "Tandem-L/ALOS-Next: A Highly Innovative Bistatic SAR Mission for Global Observation of Dynamic Processes on the Earth's Surface,” IEEE Geosci. Remote Sens. Mag., Vol. 3, 2015.

[11] F. Bordoni, M. Younis, E. Makhoul Varona, N. Gebert, G. Krieger, "Performance Investigation on Scan-On-Receive and Adaptive Digital Beam-Forming for High-Resolution Wide-Swath Synthetic Aperture Radar", ITG Workshop on Smart Antennas, pp. 114-121, 2009.

[12] M. Suess, W. Wiesbeck, "Side-Looking SAR System", patent application WO 02/075353 A1, 2001.

[13] M. Younis, T. Rommel, F. Bordoni, G. Krieger, A. Moreira, "On the Pulse Extension Loss of Digital Beamforming SAR," IEEE Geosci. Remote Sens. Letters, 2015.

[14] P. Comon, C. Jutten, Eds., "Handbook of Blind Source Separation Independent Component Analysis and Applications", Academic Press, Oxford, 2010 